\title{
Relación entre los estilos de crianza, el estado emocional de los padres, la ideación suicida y síntomas depresivos en niños de 9 a 11 años ${ }^{14}$
}

\author{
Kelly Romero Acosta \\ PhD. Psicopatología de niños, adolescentes y adultos \\ Corporación Universitaria del Caribe CECAR, Colombia \\ Correo electrónico: kelly.romero@cecar.edu.co \\ Leodanis Fonseca Beltrán \\ Psicóloga \\ Corporación Universitaria del Caribe CECAR, Colombia \\ Correo electrónico: leodanis.fonseca@cecar.edu.co \\ Salomón Verhelst Montenegro \\ Ms. en Filosofía \\ Corporación Universitaria del Caribe CECAR, Colombia \\ Correo electrónico: salomon.verhelst@cecar.edu.co \\ José Francisco Restrepo Herrera \\ Ms. En Trabajo social \\ Corporación Universitaria del Caribe CECAR, Colombia \\ Correo electrónico: jose.restrepo@cecar.edu.co
}

Recibido: 02/03/2020

Evaluado: 01/04/2020

\section{Resumen}

El presente estudio tuvo como objetivo analizar la relación entre los estilos de crianza, el estado emocional de los padres, y la ideación suicida y la depresión, en niños de instituciones educativas públicas y privadas de la ciudad de Sincelejo-Sucre, Colombia. Participaron 190 niñas (35.8\%) y 340 niños (64.2\%), y sus padres. Los resultados obtenidos demuestran que no hay riesgo de depresión en la mayoría de la muestra; el estilo de crianza más frecuente fue el autoritativo, seguido del negligente; la depresión infantil se asoció con el sexo femenino, con la depresión de los padres y con el estilo de crianza negligente, y la ideación suicida infantil se asoció significativamente con el estilo de crianza negligente y autoritario.

Palabras clave Estilos de crianza, ideación suicida, niños, niñas, sintomatología depresiva.

14 Para citar este artículo: Romero-Acosta, K., Fonseca-Beltrán, L., Verhelst-Montenegro, S., y RestrepoHerrera, J. (2021). Relación entre los estilos de crianza, el estado emocional de los padres, la ideación suicida y síntomas depresivos en niños de 9 a 11 años. Informes Psicológicos, 21(2), pp. 229-242 http://dx.doi.org/10.18566/infpsic.v21n2a14 


\section{Relationship between parenting styles, parents' emotional state, suicidal ideation and depressive symptoms in children aged 9 to 11 years}

Abstract

This study aimed to analyze the relationship between parenting styles, parents' emotional state, and suicidal ideation and depression in children from public and private educational institutions in the city of Sincelejo-Sucre, Colombia. 190 girls (35.8\%) and 340 boys (64.2\%), and their parents participated. Results show that there is no risk of depression in the majority of the sample. The most frequent parenting style was authoritative, followed by negligent. Childhood depression was associated with female sex, parental depression and negligent parenting style, and childhood suicidal ideation was significantly associated with negligent and authoritative parenting style.

Keywords

Parenting styles, suicidal ideation, boys, girls, depressive symptoms.

\section{Relação entre estillos parentais, estado emocional dos pais, ideação suicida e sintomas depressivos em crianças de 9 a 11 anos}

Resumo

0 presente estudo teve como objetivo analisar a relação entre estilos parentais, estado emocional dos pais, ideação suicida e depressão em crianças de instituições de ensino públicas e privadas da cidade de Sincelejo-Sucre, Colombia. Participaram 190 meninas (35.8\%) e 340 meninos (64.2\%) e seus pais. 0s resultados obtidos mostram que não há risco de depressão na maioria da amostra; 0 estilo parental mais frequente foi autoritário, seguido por negligente; a depressão infantil foi associada ao sexo feminino, depressão dos pais e estilo parental negligente, e a ideação suicida na infância foi significativamente associada ao estilo parental negligente e autoritário.

Palavras chave

Estilos parentais, ideação suicida, meninos, meninas, sintomas depressivos. 


\section{ntroducción}

La Organización Mundial de la Salud (OMS, 2014) define el suicidio como el acto de quitarse la vida deliberadamente; el intento suicida como cualquier comportamiento que, sin ser letal, implica el hecho de autolesionarse con o sin intención de quitarse la vida; y el comportamiento suicida como un abanico de conductas dirigidas a hacerse daño a sí mismo: va desde los pensamientos hasta las acciones suicidas propiamente dichas.

Por otra parte, Vargas y Saavedra (2012) afirman que la conducta suicida podría considerarse como una sucesión de pasos que inicia con aspectos cognitivos, como la ideación suicida, hasta aspectos conductuales, tales como el intento suicida o su consumación; con referencia a la ideación suicida sostienen que ésta incluye un amplio espectro de pensamiento que puede tomar diversas formas de presentación como el deseo de morir, la idea suicida sin llegar a planear la acción, la ideación suicida con un plan determinado o la intensa preocupación autodestructiva de naturaleza delusiva.

Las formas como se presentan las ideas suicidas también pueden variar de acuerdo a la edad. Según Silva (2019), la secuencia entre idea suicida y planificación se presenta de forma menos lineal, cuando el individuo tiene menos edad, es así que, en la infancia, la planeación puede no venir anticipada por una ideación suicida y se puede actuar sin pensar. Así mismo, la ideación suicida no necesariamente se relaciona con un concepto de muerte en todo el sentido de la palabra ("ya jamás volveré"), sino que se puede entender como la muerte en los videojuegos o las caricaturas, donde los personajes mueren, pero pueden retornar en algún momento, es decir, que el niño percibe la muerte como una situación reversible y temporal, asociada a pensamientos mágicos (Zañartu, Krämer, \& Wietstruck, 2008). En efecto, el concepto de muerte en la infancia tiene su propio desarrollo según la edad que tenga el individuo (Zañartu et al., 2008).

Por ejemplo, a los dos años, los niños que se encuentran en una etapa pre-operacional (Piaget, 1967) no tienen un concepto de muerte propiamente dicho, más bien se centran en su cuerpo, en las nuevas conductas que adquieren y cuando sienten dolor, pueden mostrar conductas de irritabilidad y de evitación. En la etapa de los tres a los siete años, ya los niños tienen un pensamiento pre-operacional, y tendrían una idea de muerte reversible. A esa muerte se le atribuyen aspectos mágicos y no es eterna, es temporal. En la etapa del pensamiento operacional, que se inicia aproximadamente a los siete años, el concepto de muerte es más evolucionado porque el niño cuenta con algunos conceptos como la universalidad o la irrevocabilidad. Sin embargo, es a partir de los doce años cuando el niño adquiere un pensamiento hipotético-deductivo; así, la muerte puede verse como algo universal y permanente, el nivel de abstracción es más complejo y el niño es consciente de que todos podemos morir y no volver, pero ¿de dónde no volvemos? La idea de la propia muerte puede aparecer a partir de los siete años y evolucionar -en algunos casos- llegando el niño o la niña a expresar: "Me quiero morir, me quiero matar, pero no lo haría". 
A propósito del suicidio en la infancia, Meinzer y Pettit (2014, citados en Covarrubias, Soltero, Sánchez, Rivera, \& Pavón, 2017) aseveran que un siglo atrás se consideraba poco probable, dada la creencia de que los niños no logran comprender este hecho, sin embargo, actualmente el suicidio infantil es una realidad. De manera similar, Cabra, Infante y Sossa (2010) coinciden en que el suicidio en población pediátrica es un fenómeno relativamente poco frecuente, pero que en las últimas décadas ha aumentado considerablemente; además, reconocen que poco se ha estudiado sobre el tema. A pesar de ello, se ha logrado establecer que existe pluralidad de factores de riesgo relacionados con el comportamiento suicida en niños, entre ellos: baja tolerancia a la frustración, dificultad para resolver conflictos, síntomas depresivos, baja autoestima, impulsividad, maltrato, disfuncionalidad familiar, antecedentes psiquiátricos, antecedentes de abuso sexual, problemas escolares, aislamiento social, búsqueda de riesgo, entre otros (Restrepo-Herrera, Romero-Acosta, \& Verhelst-Montenegro, 2019; Rivas et al., 2017; Villalobos-Galvis, Ojeda-Rosero, \& Luna-Tascón, 2019).

Partiendo de los supuestos anteriores, es fundamental reconocer, en primer lugar, que los factores de riesgo varían según aspectos individuales, ambientales y contextuales; en segundo lugar, que la vulnerabilidad del niño depende de la cantidad y cualidad de los factores presentes, así como del tiempo de exposición a ellos; y, en tercer lugar, que se debe a múltiples factores (Ramires, Passarini, Flores, \& Santos, 2009). Es difícil identificar situaciones relacionadas con la conducta suicida en niños y adolescentes, a pesar de que se ha establecido que existen ciertos factores de riesgo asociados a esta conducta. Empero, el contexto familiar y escolar son fundamentales a la hora de identificar dichos factores (Giraldo, Ospina, \& Flórez, 2018; Martínez \& Robles, 2016). Muchos niños y adolescentes comentan sus problemas en la escuela con el psicólogo escolar, pues no tienen la confianza con sus padres o no los quieren preocupar. En ocasiones pueden acercarse a un profesor elegido a quien han conferido una confianza especial. Sea como sea, es difícil lidiar con la ideación suicida y con el comportamiento suicida en niños y adolescentes; en este sentido, el papel de la escuela y de la familia puede hacer la diferencia en una situación crítica.

Con relación a la familia, González, Martínez y Ferrer (2017) señalan que la presencia de violencia intrafamiliar, la insatisfacción de los menores con su medio familiar, los métodos educativos coercitivos, la negligencia y el maltrato, tanto físico como psicológico, están directamente relacionados con el comportamiento suicida en escolares; así mismo, se han relacionado otros factores como el bullying (Carballo \& Gómez, 2017). Esto los lleva a resaltar la importancia de realizar investigaciones que profundicen en las características distintivas de la conducta suicida, a partir de enfoques preventivos, y que tengan en cuenta a la familia como grupo social esencial que interviene en el desarrollo individual de los menores. La familia, aparte de ser un grupo social fundamental, coadyuva en el proceso de aprendizaje respecto al mundo y al desarrollo emocional del individuo durante su niñez y adolescencia; dentro de este proceso el tipo de familia y el estilo de crianza y la salud mental de los padres son determinantes (Aburto, Díaz \& López, 2017). 
En relación con los estilos de crianza, se han entendido desde diferentes perspectivas teóricas; una de las primeras en hablar sobre estilos de crianza fue Baumrind (1971), quien, después de años de investigación, considera cuatro estilos de crianza principales: el autoritario, autoritativo, negligente y permisivo. También Maccoby y Martin (1983, citados en Aguilar-Yamuza, Raya-Trenas, Pino-Osuna \& Herruzo-Cabrera, 2019) plantean dos dimensiones globales que, combinadas, componen los estilos de crianza, a saber, respuesta y demanda. La respuesta incluye las manifestaciones de cariño, diálogo, contención, apoyo y calidez, mientras la demanda comprende exigencias, límites, prohibiciones, disciplina, reglas y normas. Los estilos de crianza, según esta postura, son cuatro: el autoritario, que supone una alta demanda y baja respuesta; el negligente, que se caracteriza porque tanto la demanda como la respuesta es baja; el permisivo, donde la demanda es baja y la respuesta alta; y, por último, el estilo autoritativo que está determinado por la alta demanda y la alta respuesta.

Estudios anteriores relacionan los estilos de crianza con la autoestima, el estrés psicológico, el bienestar psicosocial y la ideación suicida (Boudreault-Bouchard, Hains, Vandermeerschen \& Perron, 2013; Brassell, Rosenberg, Parent, Rough \& Seehuus, 2016). Desde otro ángulo, Pérez et al. (2013) encontraron que los estilos parentales pueden predecir la ideación suicida en población femenina y masculina adolescente; no obstante, afirman que no es la única variable que puede contribuir al desarrollo de ideación suicida, por lo que, insisten en lo importante que es ampliar la comprensión de la ideación suicida, teniendo en cuenta otras categorías de estudio. En la misma línea, Cuervo
(2010) asegura que los patrones y estilos de crianza influyen en el desarrollo socio afectivo de los niños y niñas.

Sin embargo, en Colombia no se encontró mucha información al respecto. Hay pocos estudios sobre los estilos de crianza realizados en este país (Castillo, Sibaja, Carpintero, \& Romero-Acosta, 2015). En ese sentido, es importante analizar la relación entre los estilos de crianza y la salud mental infantil, teniendo en cuenta su dualidad como factor protector o de riesgo para problemas de depresión, de conducta o de comportamiento suicida. Téngase en cuenta que el comportamiento suicida es un importante problema de salud pública a nivel mundial y que, como muy bien puntualizan Restrepo-Herrera et al. (2019), es fundamental priorizar el estudio de este fenómeno, particularmente por la situación de vulnerabilidad que viven muchas familias en Colombia a causa directa o indirecta del conflicto armado, especialmente en el departamento de Sucre, Colombia.

Habida cuenta de todo lo enunciado, el objetivo de este trabajo fue establecer la relación entre los estilos de crianza, el estado emocional de los padres, la ideación suicida y la depresión, en niños de instituciones educativas públicas y privadas de la ciudad de Sincelejo-Sucre, Colombia.

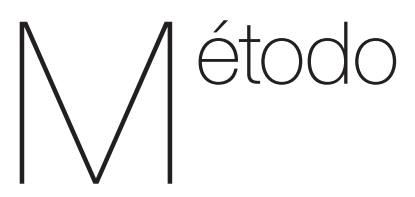

Diseño

El presente estudio se realizó bajo el paradigma cuantitativo con corte 
transversal y de tipo explicativo, es decir, se hizo medición numérica de las variables, los datos fueron recogidos en un solo momento y se midió el grado de relación de las variables en la muestra (Hernández, Fernández, \& Baptista, 2014).

\section{Participantes}

La muestra estuvo conformada por 190 niñas (35.8\%) y 340 niños (64.2\%), y sus padres. El tipo de muestreo fue por conglomerados, se escogieron los colegios de diferentes zonas de la ciudad para que se tuviera representatividad de estratos. De esos colegios, se les envío la invitación a todos los niños que asistían a clases de quinto de primaria.

Participaron más madres ( $n=437$; $82.5 \%$ ), que padres ( $\mathrm{n}=93 ; 17.5 \%$ ); la media de edad de los hijos fue de 10.15 (DS $=.477)$ y la mayoría de los colegios fueron públicos ( $n=412 ; 77.7 \%$ ). Esta información se encuentra más detallada en la Tabla 1.

Tabla 1.

Características sociodemográficas

\begin{tabular}{ccc}
\hline \multicolumn{1}{l}{ Sexo } & N & $\%$ \\
\hline Niña & & \\
Niño & 190 & 35.8 \\
\hline Sexo padres & 340 & 64.2 \\
\hline Madre & & \\
Padre & 437 & 82.5 \\
\hline Edad & 93 & 17.5 \\
\hline 9 & & \\
\hline 10 & 27 & 5.1 \\
11 & 398 & 75.1 \\
\hline
\end{tabular}

\section{Procedimiento}

En primer lugar, durante enero y febrero de 2016 se eligió un grupo de instituciones educativas al azar de una lista que brindó la Alcaldía de Sincelejo, Colombia, que contenía todos los establecimientos educativos de la ciudad. Un total de diecisiete escuelas fueron invitadas, sin embargo, solo catorce de ellas aceptaron. Los investigadores enviaron una carta de presentación que explicó los objetivos del proyecto de investigación a todos los directores de los colegios; luego, los que aceptaron participar fueron visitados personalmente y se les explicó cuidadosamente las condiciones del proyecto. Solo participaron los padres y los niños y niñas que así lo quisieron y que firmaron el consentimiento informado. A los padres se les envió la batería de pruebas a casa y a los hijos se les aplicó en el colegio, en los salones de clase o en salones alternos establecidos por los colegios. En cada aplicación se encontraba, al menos, un asistente investigador con especialización en investigación educativa y dos estudiantes de pregrado del programa de psicología de la Corporación Universitaria del Caribe, Colombia.

\section{Instrumentos}

A continuación, se detallan los instrumentos aplicados a los padres y a los niños y niñas.

\section{Instrumentos aplicados a los padres}

Beck Depression Inventory (BDI; Beck, Steer, \& Brown, 1996). El Inventario de depresión de Beck se utilizó para evaluar los síntomas depresivos de los adultos. 
El BDI-II es un cuestionario de 21 ítems que examina los síntomas cognitivos, conductuales, afectivos y somáticos de la depresión. Cada elemento del BDI-II se compone de una serie de declaraciones ordenadas por rango. A cada enunciado se le asigna un puntaje de 0 a 3 que refleja la gravedad del síntoma. Aquellos individuos que obtengan un puntaje de 14 a 19 puntos podrían tener depresión leve; de 20 a 28 puntos, moderada y de 29 a 63 puntos, severa; sin embargo, para el diagnóstico de depresión deberá aplicarse una entrevista diagnóstica propiamente dicha pues éste es un instrumento de cribado y solamente indica la posibilidad de depresión. Este instrumento ha sido el más utilizado internacionalmente para medir síntomas depresivos en muestras clínicas y no clínicas, dado que se ha demostrado su alto grado de consistencia interna (coeficiente alfa entre .78 y .92), así como buenos índices de fiabilidad (Sanz \& Vásquez, 1998). Ademas, se ha validado en población latinoamericana, resultando satisfactoria su consistencia interna con un alfa de Cronbach de .90 (Melipillán, Cova, Rincón, \& Valdivia, 2008; Estrada, Delgado, Landero, \& González, 2015). Para este estudio el Alpha de Cronbach fue de .834.

Cuestionario de datos sociodemográficos (ad hoc). Los participantes completaron un cuestionario sociodemográfico que incluyó fecha y lugar de nacimiento, género, nivel educativo y nivel educativo de los padres.

Instrumentos aplicados a los niños y a las niñas

Kutcher's Depression Scale (KADS11; Brooks, Krulewicz \& Kutcher, 2004), para evaluar la sintomatología depresiva en niños y niñas. Este instrumento es una escala de autoinforme diseñada para identificar y evaluar la gravedad de los síntomas depresivos; la versión utilizada está conformada por 11 ítems redactados en terminología estándar y las respuestas puntúan en una escala simple de 4 puntos que va de 0 a 3. Se ha demostrado que esta escala posee una consistencia interna satisfactoria, fiabilidad de .79 y tiene suficiente capacidad para diferenciar individuos deprimidos de los no deprimidos (Shahidi \& Shojaee, 2014). La ideación suicida se midió teniendo en cuenta el ítem 11 del KADS, el cual indaga sobre la ideación suicida de la siguiente manera: Has tenido pensamientos, planes o acciones para suicidarte o hacerte daño a ti mismo: a) sin pensamientos o planes o acciones; b) pensamientos ocasionales, no hay planes o acciones; c) pensamientos frecuentes, no hay planes o acciones; d) planes y / o acciones que han hecho dañado. Esta escala no ha sido validada a población colombiana, sin embargo, para este estudio, el Alpha de Cronbach fue de 893 .

Escala de Prácticas Parentales (Lempers, Clarke-Lempers, \& Simons, 1989). La escala de prácticas parentales contiene 29 ítems que indagan sobre las estrategias que usan los padres para interactuar y cuidar de ellos en los últimos seis meses. Esta escala se compone de cuatro subescalas que representan cuatro estilos parentales: autoritario, autoritativo, negligente y permisivo. En general, tiene buenas propiedades psicométricas y ha sido usada por investigadores para evaluar estilos parentales (Lempers et al., 1989). Aunque no ha sido validada a población colombiana, 
para este estudio presenta una buena consistencia interna (.790).

Cuestionario de datos sociodemográficos (ad hoc), que buscaba adquirir los siguientes datos: fecha y lugar de nacimiento, género y nivel educativo de los padres.

\section{Análisis estadístico}

Con la ayuda del Statistical Package for Social Science (SPSS v. 20) se calcularon frecuencias, porcentajes, medias y desviaciones estándar, según el caso. También se llevaron a cabo dos regresiones lineales, tomando como variables dependientes la depresión y la ideación suicida. En el modelo se incorporó la edad, el sexo, el posible riesgo de depresión de los padres y los estilos de crianza.

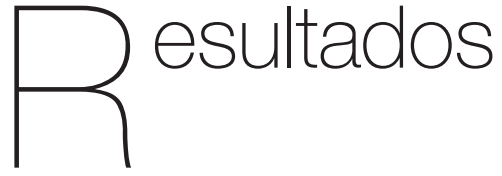

\section{Presencia de síntomas en la muestra y de ideación suicida}

Los niños obtuvieron una media de 15.43 (DS = 5.06) y los padres de 10.25 (DS = 8.70); esto quiere decir que los niveles de depresión no superaron los puntos de corte de las escalas, no hay riesgo de depresión en la mayoría de la muestra. Con respecto a la ideación suicida, 98 niños (18.5\%) han tenido pensamientos ocasionales y/o frecuentes de suicidio sin planes o acciones, mientras que 22 participantes (4.2\%) han hecho planes o realizado acciones que les han dañado. Esta información se encuentra más detallada en la Tabla 2.

Tabla 2.

Presencia de síntomas depresivos en padres y niños(as)

\begin{tabular}{cccccc}
\hline & N & Mínimo & Máximo & Media & DE \\
\hline Depresión infantil & 529 & 11.00 & 41.00 & 15.43 & 5.06 \\
\hline & \multicolumn{2}{c}{ Niños(as) } & & \\
\hline Depresión en adultos & 526 & .00 & 52.00 & 10.25 & 8.70 \\
\hline
\end{tabular}

\section{Presencia de estilos de crianza en la muestra}

El estilo de crianza más característico de la muestra fue el autoritativo (45.5\%), seguido del negligente (32.3\%) (ver Tabla 3).
Tabla 3.

Estilos de crianza de la muestra

\begin{tabular}{ccc}
\hline Tipos de estilos de crianza & N & $\%$ \\
\hline Autoritativo & 241 & 45.5 \\
Negligente & 169 & 32.3 \\
Permisivo & 73 & 13.8 \\
Autoritario & 39 & 7.4
\end{tabular}




\section{Resultado de la regresión lineal con depresión infantil como variable dependiente \\ Como se evidencia en la Tabla 4, la depresión infantil se relacionó con ser niña $(p=.29)$, con la depresión de los pa- dres $(p=.007)$ y con el estilo de crianza negligente $(p<.001)$.}

Tabla 4.

Determinantes de la depresión infantil

\begin{tabular}{lccccc}
\hline & B & 95\% CI B & B & Valor-P & R2 \\
\hline Edad participantes & .54 & $-.344 ; .729$ & .051 & .229 & .076 \\
Sexo participantes & -1.05 & $.020 ;-1.93$ & -.100 & .020 & \\
Depresión padres & .08 & $.001 ; .037$ & .148 & .001 & \\
Estilo negligente & 2.03 & $1.06 ; 3.0$ & .189 & .000 & .180 \\
Estilo autoritario & 1.14 & $-.53 ; 2.8$ & .059 & .553 & \\
Estilo permisivo & .39 & $-.90 ; 1.69$ & .027 & \\
\hline
\end{tabular}

\section{Resultado de la La Tabla 5 muestra que la ideación regresión lineal con ideación suicida como variable dependiente suicida en niños de nueve a once años se asocia significativamente con el estilo de crianza negligente $(p=.002)$ y el estilo de crianza autoritario $(p=.001)$.}

Tabla 5.

Determinantes de la ideación suicida en niños

\begin{tabular}{lccccc}
\hline & $\mathrm{B}$ & $95 \% \mathrm{Cl} \mathrm{B}$ & $\mathrm{B}$ & Valor-P & $\mathrm{R}^{2}$ \\
\hline Edad participantes & .067 & $-.076 ; .21$ & .040 & .359 & .021 \\
Sexo participantes & -.00 & $-.149 ; .134$ & -.004 & .921 & \\
Depresión padres & .00 & $-005 ; .011$ & .035 & .426 & .001 \\
Estilo negligente & .25 & $-098 ; .408$ & .149 & .001 & .421 \\
Estilo autoritario & .47 & $.207 ; .746$ & .156 & .037 & \\
Estilo permisivo & .08 & $-.122 ;, 292$ & & \\
\hline
\end{tabular}

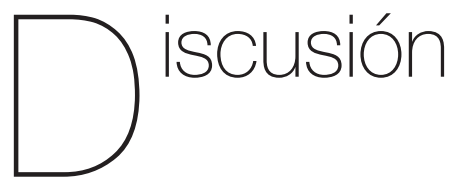

El presente estudio analizó la relación entre los estilos de crianza, el estado emocional de los padres, la ideación suicida y los síntomas depresivos en niños de nueve a doce años de la ciudad de Sincelejo (Sucre, Colombia). Los resultados obtenidos demuestran que no hay riesgo de depresión en la mayoría de la muestra y que el estilo de crianza más frecuente fue el autoritativo, seguido del negligente; asimismo, la depresión infantil se asoció con el sexo femenino, con 
la depresión de los padres y con el estilo de crianza negligente, y, por último, la ideación suicida infantil se asoció significativamente con el estilo de crianza negligente y autoritario.

El estilo de crianza que mostró mayor prevalencia fue el autoritativo (45.5\%), lo que indica que en la dinámica familiar de la mayoría de la muestra habitualmente se dialoga entre padres e hijos, hay manifestaciones de cariño, apoyo y calidez, además de que se imparte disciplina, se establecen límites, reglas y normas; otro amplio porcentaje de la muestra reportó como estilo de crianza el negligente $(32.3 \%)$, lo que se traduce en pocas manifestaciones de cariño, contención, apoyo y calidez, así como bajas exigencias, pocos límites, prohibiciones y normas.

Los resultados obtenidos demuestran que no hay riesgo de depresión en la mayoría de la muestra, lo cual es un punto positivo; sin embargo, no deja de ser importante desarrollar estrategias de intervención dirigidas a prevenir este tipo de problemas. Tal como señala Cuervo (2010), la presencia de sintomatología depresiva y el estrés de los padres puede influir negativamente en los estilos de crianza, generar agresividad, rechazo hacia los hijos y, consecuentemente, afectar su desarrollo emocional y social.

Aguilar-Yamuza et al. (2019) señalan que la presencia de síntomas depresivos tiende a relacionarse con interacciones negligentes o negativas por parte de los progenitores. Este dato coincide con los resultados hallados en la presente investigación, específicamente con la relación significativa entre el estilo de crianza negligente y la depresión infantil. Del mismo modo, la depresión infantil se asoció con pautas de crianza negligentes o interacciones negativas por parte de los progenitores (Aguilar-Yamuza et al., 2019), tal como se constata en los resultados de esta investigación.

Es preciso señalar que, en línea con investigaciones anteriores (Vásquez, 2013; Esparza \& Rodríguez, 2009; Zolog et al., 2011), ser niña se asoció a la presencia de síntomas depresivos, esto podría tenerse en cuenta a la hora de identificar casos de riesgo de depresión en atención primaria o en el ámbito escolar. Tanto los docentes como el personal de atención primaria debería aplicar escalas de cribado de síntomas depresivos en niñas, esto podría evitar el desarrollo del trastorno propiamente dicho, ya que, cuanto más temprano se diagnostiquen los síntomas depresivos o el riesgo a sufrir depresión infantil, más posibilidades hay de evitar que evolucione a un problema mayor. De igual modo, los hallazgos indican que los estilos de crianza negligente y autoritario se correlacionan significativamente con la ideación suicida en niños y niñas. Al respecto, Sarmiento y Aguilar (2011) coinciden en que el estilo de crianza negligente constituye un predictor de ideación suicida tanto para el sexo masculino como para el femenino. Se ha planteado también que la negligencia parental predice la presencia de psicopatología, específicamente, se ha observado que el rechazo por parte de los padres incrementa el riesgo de suicidio (Morales et al., 2014).

No solo el estilo de crianza negligente se asocia a problemas emocionales, ya que el estilo de crianza autoritario predice depresión mayor, problemas de autoestima e ideación suicida en el adolescente 
(Aburto et al., 2017). Aunque en el presente estudio el estilo autoritario no se asoció con la depresión, sin embargo, sí se asoció con la ideación suicida.

Finalmente, Castillo et al. (2015) sugirieron ampliar los estudios en las diferentes regiones de Colombia sobre estilos de crianza, realizar investigaciones que tuvieran en cuenta a los padres e investigar la asociación entre los estilos de crianza y la presencia de problemas psicológicos. La presente investigación constituye un aporte significativo dentro de la búsqueda de respuestas a estos planteamientos, dado que el estudio se realizó en el Caribe Colombiano, se estudiaron los estilos de crianza y se observó su relación con otras variables que constituyen problemas de salud pública en el país.

\section{Limitaciones del estudio}

En primer lugar, éste es un estudio transversal, por lo tanto, no se pueden hacer inferencias causales entre las variables. En segundo lugar, participaron más varones que féminas, por lo que se deben evitar generalizaciones sobre los resultados. En tercer lugar, la R2 de los modelos explicativos de la depresión y de la ideación suicida infantil son bajas, consideramos pertinente no generalizar los datos y llevar a cabo más investigaciones que confirmen o rechacen los modelos. No obstante, a pesar de esta limitación, se trata del primer estudio con estas características en el departamento de Sucre (Colombia).

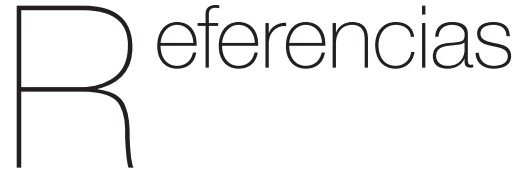

Aburto, C., Díaz, K., \& López, P. (2017). Ideación suicida en adolescentes del área rural: estilos de crianza y bienestar psicológico. Revista Colombiana de Enfermería, 15, 50-61.

Aguilar-Yamuza, B., Raya-Trenas, A., PinoOsuna, M., \& Herruzo-Cabrera, J. (2019). Relación entre el estilo de crianza parental y la depresión y ansiedad en niños entre 3 y 13 años. Revista de psicología clínica con niños y adolescentes, 6(1), 36-43.

Baumrind, D. (1971). Current patterns of parental authority. Developmental Psychology Monograph, 4, 1-103.

Baumrind, D. (1996). Parenting: The discipline controversy revisited. Family Relations, 45, 405-161.

Beck, A. T., Steer, R. A., \& Brown, G. K. (1996). BDI-Il, Beck Depression Inventory: Manual. New York: Harcourt Brace.

Boudreault-Bouchard, A.-M., Hains, J., Vandermeerschen, J., \& Perron, M. (2013). Impact of parental emotional support and coercive control on adolescents' selfesteem and psychological distress: results of a four-year longitudinal study. Journal of Adolescence, 36(4), 695-704.

Brassell, A., Rosenberg, E., Parent, J., Rough, J., \& Seehuus, M. (2016). Parent's psychological flexibility: associations with parenting and child psychosocial wellbeing. Journal of Contextual Behavioral Science, 5(2), 111-120. 
Brooks, S. J., Krulewicz, S. P., \& Kutcher, S. (2004). The Kutcher Adolescent Depression Scale: Assessment of Its Evaluative Properties over the Course of an 8-Week Pediatric Pharmacotherapy Trial. Journal of Child and Adolescent Psychopharmacology, 13, 337-349.

Cabra, O., Infante, D., \& Sossa, F. (2010). El suicidio y los factores de riesgo asociados en niños y adolescentes. Revista Médica Sanitas, 13(2), 28-35.

Carballo, J., \& Gómez, J. (2017). Relación entre el bullying, autolesiones, ideación suicida e intentos autolíticos en niños y adolescentes. Revista de Estudios de Juventud, (115), 207-218.

Castillo, S., Sibaja, D., Carpintero, L., \& Romero-Acosta, K. (2015). Estudio de los estilos de crianza en niños, niñas y adolescentes en Colombia: Un estado del arte. Busqueda, 2(15), 64-71.

Covarrubias, M., Soltero, R., Sánchez, L., Rivera, M., \& Pavón, D. (2017). Pensamientos de muerte e ideación suicida en niños y niñas mexicanos de 6 a 11 años. UARICHA Revista de Psicología, 14(32), 20-30.

Cuervo, A. (2010). Pautas de crianza y desarrollo socioafectivo en la infancia. Diversitas: Perspectivas en Psicología, 6(1), 111-121.

Esparza, N., \& Rodríguez, M. (2009). Factores contextúales del desarrollo infantil y su relación con los estados de ansiedad y depresión. Diversitas: Perspectivas en Psicología, 5(1), 47-65.

Estrada, B., Delgado, C., Landero, R., \& González, M. (2015). Propiedades psicométricas del modelo bifactorial del BDI-II (versión española) en muestras mexicanas de población general y estudiantes universitarios. Universitas Psychologica, 14(1), 125-136.

Giraldo, L., Ospina, S., \& Flórez, J. (2018). Efectos emocionales del intento de suicidio de una niña de 10 años en los familiares con quienes reside en la ciudad de Medellín durante 2015-2016. Poiésis, (35), 108-119.

González, R., Martínez, L., \& Ferrer, D. (2017). Funcionamiento familiar e intento suicida en escolares. Revista Cubana de Medicina General Integral, 33(3), 281-295.

Hernández, S., Fernández, C., \& Baptista, P. (2014). Metodología de la investigación. México D.F. , México: Mc Graw Hill Education.

Jorge, E., \& González, M. (2017). Estilos de crianza parental: una revisión teórica. Informes psicológicos, 17(2), 39-66.

Lempers, J., Clarke-Lempers, D., \& Simons, R. (1989). Economic hardship, parenting, and distress in adolescence. Child Development, 60, 25-39.

Martínez, J., \& Robles, A. (2016). Percepción de actores sociales sobre la conducta suicida: Analisis de contenido a través de grupos focales. Informes Psicológicos, 16(2), 53-68.

Melipillán, R., Cova, F., Rincón, P., \& Valdivia, M. (2008). Propiedades psicométricas del inventario de depresión de BeckII en adolescentes Chilenos. Terapia Psicológica, 26(1), 59-69. 
Morales, S., Armijo, I., Moya, C., Echávarri, O., Barros, J., Varela, C., . . S Sánchez, M. (2014). Percepción de cuidados parentales tempranos en consultantes a salud mental con intento e ideación suicida. Avances en Psicología Latinoamericana, 32(3), 403-417.

Organización Mundial de la Salud (OMS). (2014). Preventing Suicide. Luxemburgo: OMS.

Pérez, A., Uribe, J., Vianchá, M., Bahamón, M., Verdugo, J., \& Ochoa, S. (2013). Estilos parentales como predictores de ideación suicida en estudiantes adolescentes. Psicología desde el Caribe, 30(3), 551-568.

Piaget, J. (1967). El lenguaje y el pensamiento en el niño. Barcelona: Paidos.

Pineda, D., Valiente, R., Martínez-Martínez, A., Chorot, P., \& Sandín, B. (2017). Síntomas ansioso-depresivos en niños y su relación con los estilos educativos de los padres. Información Psicológica, 114, 73-82.

Ramires, R., Passarini, S., Flores, G., \& Santos, G. (2009). Fatores de risco e problemas de saúde mental de crianças. Arquivos Brasileiros de Psicologia, 61(2), 1-14.

Restrepo-Herrera, J., Romero-Acosta, K., \& Verhelst-Montenegro, S. (2019). Caracterización del Suicidio en el departamento de Sucre: Un análisis de las noticias publicadas en el Periódico El Meridiano de Sucre, Colombia. Búsqueda, 6(22), 1-14.

Rivas, K., Yanez, V., Ramírez, A., Díaz, D., Bueso, O., Godoy, C., \& Barahona, J. (2017). Variables sociodemográficas y psicosociales del intento suicida en pacientes pediátricos, Hospital Escuela
Universitario, Tegucigalpa 2012-2015. CIMEL, 22(1), 11-20.

Santos, R., \& Melo, M. (2016). Tendencia suicida en niños accidentados. Psicología: Ciência e Profissão, 36(3), 571-583.

Sanz, J., \& Vásquez, C. (1998). Fiabilidad, validez y datos normativos del inventario para la depresión de Beck. Psicothema, 10(2), 303-318.

Sarmiento, C., \& Aguilar, J. (2011). Predictores familiares y personales de la ideación suicida en adolescentes. Psicología y Salud, 21(1), 25-30.

Shahidi, M., \&Shojaee, M. (2014). Psychometric properties and diagnostic utility of the 11-item kutcher adolescent depression scale (KADS-11) in Persians Samples. International Journal of Psychology and Behavioral Sciences, 4(6), 201-207.

Silva, L. (2019). Suicide among children and adolescents: a warning to accomplish a global imperative. Acta Paulista de Enfermagem, 32(3), 3-6.

Vargas, H., \& Saavedra, J. (2012). Prevalencia y factores asociados con la conducta suicida en adolescentes de Lima Metropolitana y Callao. Revista Peruana de Epidemiología, 16(3), 1-11.

Vásquez, A. (2013). Depresión. Diferencias de género. Multimed, 17(3), 193-217.

Villalobos-Galvis, F., Ojeda-Rosero, E., \& LunaTascón, E. (2019). Caracterización de las conductas suicidas en adolescentes de la zona de influencia del volcán Galeras, Nariño, Colombia. Informes Psicológicos, 19(2), 163-180. 
Zañartu, C., Krämer, C., \& Wietstruck, M. Zolog, T., Jané, M., Bonillo, A., Canals, (2008). La muerte y los niños. Revista Chilena de Pediatría, 79(4), 393-397.

J., Hernández, C., Romero-Acosta, K., \& Doménech-Llaberia, E. (2011). Age, gender and negative life events in anxiety and depression self-reports at preadolescence and early adolescence. Ansiedad y Estrés, 17(17), 113-124. 